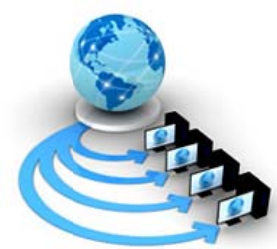

Volume 9, No. 2, March-April 2018

\title{
A COMPARATIVE STUDY OF OBJECT TRACKING ALGORITHMS AND A PROPOSED WORK ON AN OBJECT TRACKING QUADCOPTER
}

\author{
Yash Shah, \\ NMIMS,Mumbai, India. \\ Prakhar Tripathi, \\ NMIMS,Mumbai, India.
}

\author{
Amitesh Upadhyay, \\ NMIMS,Mumbai, India. \\ Prof. Artika Singh, \\ Computer Engineering Dept., NMIMS, \\ Mumbai, India.
}

\begin{abstract}
Nowadays, the development of Unmanned Aerial Vehicle (UAV) is a huge field of research and researchers want to explore the application. There are a huge number of projects and research topics emerging in this field. Research has shown that quadcopter is the most accomplished and easy to construct UAV. Here, we present a comparative study on object tracking algorithms to decide which algorithm would be most suitable for integrating in an autonomous quadcopter.
\end{abstract}

Keywords: ZEUS; UAV; PID; PHANTOM; IRLOCK; KLT; TLD; Camshift and Mean-Shift

\section{INTRODUCTION}

With recent advancements in robotics and artificial intelligence, many inexpensive robots, such as AR. Drone, have surfaced, with various sensors and actuators. Tasks performed by these robots include object tracking and following. In a video sequence, an object is said to be in motion if it is changing its location with respect to its background. Motion tracking is actually the process of keeping track of that moving object in the video sequence, i.e. position of a moving object at a certain time [6]. The idea is that in a snapshot taken by the drone's front camera, the user marks an area as an object of interest and then the drone autonomously follows this selected object as the object moves. Moreover, the software is supposed to control the drone in a dynamic environment with possible external forces, e.g., small turbulences etc.

Object tracking and following drones are becoming popular by the day. Drones such as 3DR SOLO, EHang Ghost Drone 2.0, DJI Phantom 4, Monoprice Hubsan 501S etc. are some of the most popular object following drones that are readily available in the market today[5]. These drones use various different techniques and algorithms for tracking and following any object.

Object tracking has many uses apart from simple user following. These include applications in cinematography, visual surveillance, traffic information, and mobile robots. The problems that hinder the smooth tracking include temporal variation, dynamic environment, abrupt object or camera motion, multi-camera, multi-object, computational expense and limited memory etc. Various techniques exist for tracking an object such as template matching, color detection, feature detection and matching, motion estimation, Kanade-LucasTomasi(KLT), IR-LOCK and Tracking Learning Detection(TLD).
We reviewed and compared some of the existing tracking methods and we propose to use the object tracking technique called Tracking-Learning Detection (TLD) for tracking the object on the video stream [1]. This method combines advantages of object tracking and detection which is critical because the object of interest can easily disappear from the drone's view (hence we need object detection) while the tracking method can capture different views of the object that can be used to teach the detector. The output of TLD, together with its distance is then fed to a classical proportionalintegral-derivative (PID) controller that tells the drone where to fly [1]. PID, generally used in industrial automation systems, is a closed loop feedback control system. The main job of the PID is to calculate the speed of the motors required to keep the drone stable or move in the desired direction with the desired speed.

The motivation for this review is to compare and summarize the existing techniques to be able to develop better, more efficient methods for progress and development in the said field.

\section{RELATED WORK}

Drones currently have huge number of features. Some of them being returning to launch position, stabilizing itself in case of wind (Gyro-Stabilization), Onscreen Real-Time Flight Parameters, GPS path set and flying on that path, FPV (First Person View) Drone Technology[2]. There are several different drones that are already present in this field which use several different methods. One of the main feature of current drone is object tracking [4].some of the methods to implement them are:

\section{A) Infrared Lock}

IRLock is a method not an algorithm that is used to track a particular light object. Here, we use an IR-LED. The custom- 
manufactured IR-LOCK filter enables simple and accurate target tracking for Drones [3]. Positions of objects are accurately reported with the help of IR-LED that has a detection range of about $50 \mathrm{ft}$. The IR-LOCK firmware scans the location of all IR targets, or tracks the largest IR target. The key benefit of using IR LEDs as targets is that the light of IR-LED is visible only on camera so the performance is not dependent on ambient lighting conditions (e.g., shade, dim lighting, bright sunlight, etc.) [3]. This is also the greatest disadvantage as in good lighting condition, even in 0 brightness of the software, there might be some light source detected to which the drones can change direction. This type of light source can be the sun, high powered lights or other sources of Infrared emission that may momentarily collide with the one which is being tracked.

B) Kanade-Lucas-Tomasi (KLT)

The Kanade-Lucas-Tomasi (KLT) feature tracker is an algorithm for feature extraction. It is proposed mainly to deal with the expensive costing of traditional image registration techniques [1]. KLT uses the spatial intensity information to direct the search for the position that yields the best match. It is faster than traditional techniques as it examines extremely less number of matches between the images. It basically tries to find the shift in an interest point that might have been chosen. The framework is based on a squared distance criterion over a local region that is optimized with respect to the transformation parameters, e.g. displacement in $\mathrm{x}$ and $\mathrm{y}$ axis [4]. In order to solve this problem, approximation of the feature displacement with a linear term using Taylor series is done. This algorithm is expected to work well for corner-like features that do not suffer from any aperture problem.

\section{C) CamShift \& Mean-Shift:}

CamShift is a tracking algorithm, which is derived from Mean-Shift algorithm, where it applies Mean-Shift in every single frame of a video, and records the results.

\section{CamShift algorithm includes these three parts:}

\section{Back Projection}

It is a method that uses the histogram of an image to show up the probabilities of colors that may appear in each pixel[7].

\section{Mean-Shift}

Mean-Shift is an algorithm which finds modes in a set of data samples representing an underlying probability density function (PDF) in $\mathrm{R}^{\wedge} \mathrm{N}$. It is a nonparametric clustering technique which does not require prior knowledge of the number of clusters, and does not constrain the shape of the clusters [7].

\section{Track}

Mean-Shift algorithm is used on every single frame and can be tracked by following the initial frame that is just the output window of the previous frame. This can be applied on a video obtained or frames obtained from a web camera[7].

\section{A. Inference}

In above related work, we discussed about the different methods that have to be implemented for object tracking. All these have several limitations. But still are widely used methods for object tracking.

\section{PROPOSED WORK}

The purpose of the work is to make a quadcopter able to maneuver automatically and follow an object without having to control it manually. The quadcopter movement and control will be carried out by the Arduino. The command signal will be transmitted wirelessly from the trans-receiver of Android (Bluetooth) to the trans-receiver of the quadcopter side and the quadcopter will move accordingly. The quadcopter will also include features like height variation, camera (Photography and Videography), navigation, follow me technology. All these features will also be controlled by the Arduino board. We propose to use the already available object tracking technique called Tracking-Learning Detection (TLD) for tracking the object on the video stream [1]. TLD is an algorithm for tracking of unknown objects in unconstrained video streams. The object of interest is defined by a bounding box in a single frame. TLD simultaneously tracks the object, learns its appearance and detects it whenever it appears in the video. openTLD is a software available publicly by a repository on Github that will help us achieve it.

The output of TLD, together with its distance will be fed to a classical proportional-integral-derivative (PID) controller that tells the drone where to fly. By using a PID controller, it should be feasible for our quadcopter to calculate the Roll, Pitch, and Yaw and vary each motor speed to balance the quadcopter. Using PID is favorable as it promises smoothness, performance and accuracy.

\section{CONCLUSION}

The main purpose of our project is to build a complete working model of Quadcopter with improvement in its flight duration and modes of video capturing[2]. Our main goal is to build a Quadcopter that can be used for several applications like photography enthusiasts as well as adventurers who like to explore new places [8]. With the help of the reviewed research papers, we now have the knowledge to successfully build this project. We chose a quadcopter for our project because of its accomplishments and high research opportunities. This project can go further in a variety of research work to integrate various technologies with quadcopters to get various useful outputs [8]. For this purpose, we have chosen to use Tracking Learning Detection (TLD) [1].

\section{ACKNOWLEDgment}

The above work is a combined effort of many people, knowledge from various sources and guidance from our teachers. We would like to extend a sincere thanks to Prof. Artika Singh, who provided us insight and expertise that greatly assisted us in writing this review. Many websites and research papers were taken into consideration over the study period. We would love to express our gratitude to everyone who have helped us directly or indirectly. 


\section{REFERENCES}

[1] Roman bart'ak and adam vy`skovsk'y, "Any object tracking and following by a flying drone”, 2015 Fourteenth Mexican International Conference on Artificial Intelligence.

[2] Andrey Giyenko, Young Im Cho, "Intelligent Unmanned Aerial Vehicle Platform for Smart Cities”,2016, Gachon University, Seongnam, South Korea.

[3] Aislan Gomide Foina, Raja Sengupta, Patrick Lerchi, Zhilong Liu and Clemens Krainer, "Drones in Smart Cities: Overcoming Barriers through Air Traffic Control Research”, unpublished.

[4] Fahd Rafi, Saad Khan, Khurram Shaiqq and Mubarak Shah, "Autonomous Target Following by Unmanned Aerial Vehicles”, University of Central Florida, Orlando FL, USA.
[5] Vinod Ingale, Umar Mulani, “QuadCopter Controlling Using Android Mobile Devices”, Vol. 3, Issue 6, June 2015, International Journal of Innovative Research in Computer and Communication Engineering, pp. 5856-5864.

[6] Mr. Kalpesh N. Shah , Mr. Bala J. Dutt , Hardik Modh, "Quadrotor - An Unmanned Aerial Vehicle", Vol. 2, Issue 1, 2014, International Journal of Engineering development and research, pp. 1299-1303.

[7] Eric Yuan, "Continuously Adaptive Mean Shift", http://www.eric-yuan.me/continuously-adaptive-shift/, 2013.

[8] Saurin Mukundbhai Sheth, Dhaval Patel,"Design of Quadcopter in Reconnaissance” ,2013, At GCET, Volume: 1, International Conference on Innovations in Automation and Mechatronics Engineering (ICIAME2013). 